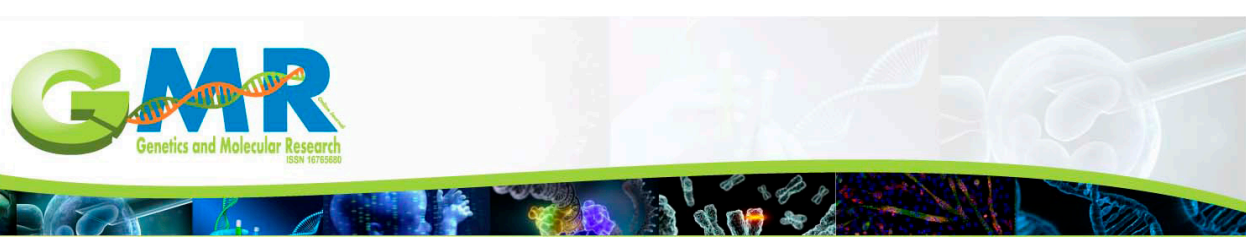

\title{
Exome sequencing in Thai patients with familial obesity
}

\author{
S. Kaewsutthi ${ }^{1}$, J. Santiprabhob ${ }^{2}$, B. Phonrat $^{3}$, A. Tungtrongchitr ${ }^{4}$, \\ P. Lertrit ${ }^{5}$ and R. Tungtrongchitr ${ }^{1}$
}

${ }^{1}$ Department of Tropical Nutrition and Food Science, Faculty of Tropical Medicine, Mahidol University, Bangkok, Thailand ${ }^{2}$ Division of Endocrinology and Metabolism, Department of Pediatrics, Faculty of Medicine Siriraj Hospital, Mahidol University, Bangkok, Thailand ${ }^{3}$ Department of Clinical Tropical Medicine, Faculty of Tropical Medicine, Mahidol University, Bangkok, Thailand

${ }^{4}$ Department of Parasitology, Faculty of Medicine Siriraj Hospital, Mahidol University, Bangkok, Thailand

${ }^{5}$ Department of Biochemistry, Faculty of Medicine Siriraj Hospital, Mahidol University, Bangkok, Thailand

Corresponding authors: R. Tungtrongchitr / P. Lertrit

E-mail: rungsunn.tun@mahidol.ac.th / patcharee.ler@mahidol.ac.th

Genet. Mol. Res. 15 (2): gmr.15028311

Received August 8, 2015

Accepted November 26, 2015

Published July 15, 2016

DOI http://dx.doi.org/10.4238/gmr.15028311

ABSTRACT. Obesity is a major worldwide health issue, with
increasing prevalence in adults and children from developed and
developing countries. Obesity causes several chronic diseases,
including cardiovascular and respiratory diseases, osteoarthritis,
hypertension, stroke, type II diabetes, obstructive sleep apnea, and
several types of cancer. Previous genome-wide association studies
have identified several genes associated with obesity, including $L E P$,
$L E P R, P O M C, P C S K 1, F T O, M C 3 R, M C 4 R, G N P D A 2, T M E M 18$,
QPCTL/GIPR, BDNF, ETV5, MAP $2 K 5 / S K O R 1, S E C 16 B$, SIM1, and
$T N K S / M S R A$. However, most of these variants are found in the intronic 
or intergenic regions, making it difficult to elucidate the underlying mechanisms. Therefore, in this study, we performed a whole exome sequencing of the protein-coding regions in the total genome (exome) of two obese and one normal subject belonging to the same Thai family to identify the genes responsible for obesity. We identified 709 functional variants that were differentially expressed between obese and normal subjects; of these, 65 were predicted to be deleterious to protein structure or function. The minor allele frequency of 14 of these genes (ALOX5AP, COL9A2, DEFB126, GDPD4, HCRTR1, MLL3, OPLAH, OR4C45, PRIM2, RXFP2, TIGD6, TRPM8, USP49, and ZNF596) was low, indicating causal variants that could be associated with complex traits or diseases. Genotyping revealed HCRTR1, COL9A2, and TRPM8 to be associated with the regulation of feeding behavior and energy expenditure. These genes constituted a network of pathways, including lipid metabolism, signaling transduction, immune, membrane transport, and gene regulation pathways, and seemed to play important roles in obesity.

Key words: Exome sequencing; Variants; Gene; Obesity; Thailand; Body mass index

\section{INTRODUCTION}

Obesity is a major problem affecting the world population. The prevalence of obesity has increased worldwide, in both developed and developing countries and adults and children. The rate of obesity in preschool children has increased from 4.2 to $6.7 \%$ between 1990 and 2010. In fact, this rate has been expected to reach $9.1 \%$ by 2020 (de Onis et al., 2010). The Department of Health at the Ministry of Public Health, Thailand, has reported an increase in the percentage of school-aged obese children (6-13 years) from 13 to 17\% between 2003 and 2012. An increasing trend has also been observed in adults: the percentage of women and men with obesity has increased from 34.4 to $40.7 \%$ and 22.5 to $28.4 \%$ between 2003 2012. Obesity is a medical condition caused by the imbalance between energy intake and expenditure; this leads to the accumulation of excess body fat, and subsequently, an increase in the body mass. Obesity increases the rate of morbidity and mortality, and can lead to the development of chronic diseases, including cardiovascular and respiratory diseases, osteoarthritis, hypertension, stroke, type II diabetes, obstructive sleep apnea, and several types of cancer (Haslam and James, 2005).

Childhood obesity is a more serious issue than adult obesity, as the number, as well as size, of adipose tissues increases in obese children, while only increasing in size in adults. Obese children, therefore, end up with a greater number of adipose tissues with advancing age, resulting in adverse effects, including chronic conditions such as high cholesterol, high blood pressure, and high glucose levels, and increased difficulty in reducing weight. Therefore, there is a high probability of an obese child becoming an obese adult with a high risk of cardiovascular disease, high blood pressure, diabetes, gall bladder stones, cancer, and a shorter life span (Maffeis and Tatò, 2001).

Obesity is defined by the body mass index (BMI) of a person. A higher BMI increases 
the risk of morbidity and mortality, and adversely affects the balance between energy intake and expenditure. Environmental and genetic factors are the two main contributors to obesity. Excess food intake and lack of exercise are the most common environmental factors; moreover, lifestyle, societal pressures, and the economy are some of the factors contributing to the increase in the rate of obesity. On the other hand, individuals with a high genetic risk of obesity are more responsive to obesity despite eating a lesser quantity of food. Therefore, the identification of genes associated with increased susceptibility to obesity could provide information that could predict the disease mechanism, in turn reducing the morbidity and mortality rate of this disease.

Previous studies have identified a correlation between several genes, such as $L E P$, LEPR, POMC, PCSK1, FTO, MC3R, MC4R, GNPDA2, TMEM18, QPCTL/GIPR, BDNF, ETV5, MAP2K5/SKOR1, SEC16B, SIM1, TNKS/MSRA, and obesity, using a candidate gene approach or a genome-wide association study (GWAS) (Farooqi et al., 2003; Begriche et al., 2011; Dubern and Clement, 2012; Mul et al., 2012; Tsou et al., 2012). Most of these variants were in the intronic region; therefore, the mechanism leading to the development of disease could not be determined (Dorajoo et al., 2012; Loos, 2012). Exome sequencing is an improved version of next generation sequencing, used to identify causal variants in the functional/coding region of the gene in an efficient, time-saving, and cost-effective manner. The aim of this study was to search for candidate genes, and predict the proteins, responsible for familial early-onset obesity in a Thai family using exome sequencing.

\section{MATERIAL AND METHODS}

\section{Study subjects}

The pedigree of all patients was analyzed, and the patients were categorized by a pediatric endocrinologist at the Department of Pediatrics in the Division of Endocrinology and Metabolism (Faculty of Medicine Siriraj Hospital) at Mahidol University, Bangkok, Thailand. This obese family had several exceedingly overweight members spread over 2-3 generations. Obese and normal subjects were categorized based on the BMI (obese BMI $\geq$ $25 \mathrm{~kg} / \mathrm{m}^{2}$; normal BMI $<25 \mathrm{~kg} / \mathrm{m}^{2}$ ). Patients with Cushing syndrome, hypothyroidism, and pseudo-hypoparathyroidism, or those on drugs causing obesity, such as corticosteroids, were excluded. These subjects displayed no sign of any genetic diseases, such as Prader-Willi syndrome, Bardet-Biedl syndrome, Cohen syndrome, or MOMO syndrome. This study was approved by the Siriaj Institutional Review Board and Ethics Committee of the Faculty of Tropical Medicine, Mahidol University, Bangkok, Thailand. Informed consent was obtained from all participants.

\section{Assessment of nutritional status}

The nutritional status of all included subjects was estimated by anthropometric measurements of the weight, height, arm-circumference, waist-circumference, hipcircumference, skinfold thickness, pulse, and blood pressure, using standard procedures. The BMI was calculated based on the weight and height using the standard formula: BMI = weight $(\mathrm{kg}) /[\text { height }(\mathrm{m})]^{2}$. Body fat percentage was estimated using the bioelectric impedance measure. 


\section{Blood sample collection}

Blood samples were obtained after a 10-h fast (no food or drink, except water). Venous blood $(16 \mathrm{~mL})$ was drawn from all subjects and family members. Blood samples were divided into 2 tubes: $10 \mathrm{~mL}$ was stored in a tube containing EDTA anticoagulant for subsequent DNA extraction and the remaining $6 \mathrm{~mL}$ was used for biochemical measurements. The latter was equally divided into 3 parts $(2 \mathrm{~mL}$ each) and stored in tubes containing sodium fluoride, EDTA, and lithium heparin, in order to determine the fasting blood glucose level; HbA1C level; and the insulin, triglyceride, high-density lipoprotein cholesterol (HDL-C), low-density lipoprotein cholesterol (LDL-C), and cholesterol contents, respectively.

\section{Biochemical measurements}

Blood samples collected for the measurement of the fasting blood glucose, HbA1C, insulin, cholesterol, HDL-C, LDL-C, and triglyceride levels were analyzed at the Central Laboratory of the Department of Clinical Pathology, Faculty of Medicine Siriraj Hospital, using Cobas Integra 800 (Roche Diagnostics, Basel, Switzerland).

\section{DNA extraction}

Total DNA was extracted from peripheral leukocytes using a standard phenol/ chloroform method (Davis et al., 1986) as follows: red blood cells were lysed using an RBC lysis buffer; subsequently, the blood was centrifuged and the supernatant removed. This step was repeated several times until a clear supernatant was obtained. Thirty microliters of $20 \mathrm{mg} /$ $\mathrm{mL}$ proteinase $\mathrm{K}$ (Invitrogen ${ }^{\mathrm{TM}}$, Carlsbad, CA, USA) and $250 \mu \mathrm{L}$ lysis buffer were added for protein digestion. The protein was precipitated using phenol (Merck, Darmstadt, Germany) and chloroform (Merck). DNA was precipitated using absolute ethanol (Merck), recovered by centrifugation, and washed with $70 \%$ ethanol; the DNA pellet was dried and resuspended in distilled water. Purity of the extracted DNA was measured using a Nanophotometer, and calculated by the ratio of absorbance at $260 \mathrm{~nm}$ to that at $280 \mathrm{~nm}$.

\section{Exome sequencing}

Three samples [2 obese (Ob1_6 and Ob1_15) and 1 normal (Ob1_7)] were selected for total exome sequencing. All subjects were selected from the same generation and family to distinguish causal genes. The BMI of the two obese and one normal subjects were 38.34 , 44.29 (extreme obesity), and $<23 \mathrm{~kg} / \mathrm{m}^{2}$, respectively. Exome sequencing was performed at Macrogen Inc., Korea, using Genome Analyzer II (Illumina Inc., San Diego, CA, USA). Base calling was presented by the Illumina pipeline, using default parameters.

All exons were captured by using the SureSelect Human All Exon V4 (Agilent Technologies, Carlsbad, CA, USA) according to the manufacturer protocols. The captured DNA was sequenced on the Illumina HiSeq 2000 platform (Illumina Inc.). Parallel sequenced data were assembled using the UCSC Genome Browser on February 2009 (GRCh37/hg19) (http://genome.ucsc.edu/) and mapped by BWA (http://bio-bwa.sourceforge.net/). Variants and indels were detected using SAMTOOLS (http://samtools.sourceforge.net/). All variants were compared to those listed in dbSNP135 and the 1000 genomes project. 


\section{Selection of variants and protein prediction}

Variants observed in both obese subjects were identified, and those presented by the normal control also were removed using the R software. Functional variants (non-synonymous, frameshift_insertion, frameshift_deletion, stop_gain, stop_loss, splicing, and unknown) were selected for further analysis. Deleterious variants were selected using the freely available web services PolyPhen-2, SIFT, and VarioWatch. The selected variants were genotyped in 12 obese and 5 normal individuals of this family, by Sanger sequencing. The primers used are listed in Table 1. The data were statistically analyzed using the Fisher exact test, on the SPSS v.15 software platform (SPSS Inc., Chicago, IL, USA); $\mathrm{P}<0.05$ indicated a statistically significant difference. The possible biochemical pathway of the protein was predicted by the Reactome Pathway Browser, Kyoto Encyclopedia of Genes and Genomes (KEGG) pathway database, and the pathcards ${ }^{\text {alpha }}$ function in GeneCards ${ }^{\mathbb{B}}$.

\begin{tabular}{|c|c|c|c|c|}
\hline \multicolumn{2}{|l|}{ Gene } & Position & Primer sequence $\left(5^{\prime} \rightarrow 3^{\prime}\right)$ & Product size \\
\hline \multirow[t]{2}{*}{$A L O X 5 A P$} & Forward & $31287545-31287564$ & GTCTGGGGTGTGTTTGGTCT & \multirow[t]{2}{*}{697} \\
\hline & Reward & $31288222-31288241$ & TGCAAGGCAGAGAGTTCCTT & \\
\hline \multirow[t]{2}{*}{ COL9A2 } & Forward & $40773496-40773516$ & TGCAGCTGTCTCAGAGGAAC & \multirow[t]{2}{*}{615} \\
\hline & Reward & $40772902-40772921$ & TGGTCAGATCCCATTTAGCC & \\
\hline \multirow[t]{2}{*}{ HCRTRI } & Forward & $32092665-32092684$ & GGCTTCAGTCCTGGGTTTCT & \multirow[t]{2}{*}{675} \\
\hline & Reward & $32093320-32093339$ & GGTGGGAGGTCAGCAGATAA & \\
\hline \multirow[t]{2}{*}{$R X F P 2$} & Forward & $32360364-32360383$ & GACTCATACGGCCCTGTTGT & \multirow[t]{2}{*}{660} \\
\hline & Reward & $32361004-32361023$ & GCCACATGAACCCAGTTTTC & \\
\hline \multirow[t]{2}{*}{$\overline{S P T B}$} & Forward & $65249356-65249376$ & GCCTTACTGACCTCCTCTTGG & \multirow[t]{2}{*}{598} \\
\hline & Reward & $65248779-65248798$ & ATGGGGGAGGGTAGGAGAAG & \\
\hline \multirow[t]{2}{*}{ TRPM8 } & Forward & $234854206-234854226$ & AAGAGCACAGGGCAATGAAC & \multirow[t]{2}{*}{647} \\
\hline & Reward & $234854833-234854852$ & GGCAGAAGCATTCCTGATGT & \\
\hline
\end{tabular}

\section{RESULTS}

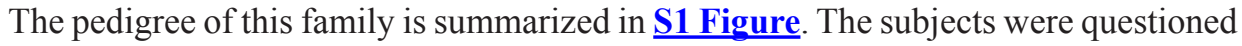
about their relatives and their eating habits over the past three generations. Seventeen [12 obese $\left(\mathrm{BMI} \geq 25 \mathrm{~kg} / \mathrm{m}^{2}\right)$ and 5 normal $\left(\mathrm{BMI}<25 \mathrm{~kg} / \mathrm{m}^{2}\right)$ subjects] DNA samples were collected from this family. The transmission of causal obesity genes was surmised to follow an autosomal dominant pattern, as obese members were found in every generation (black circle or square). Two obese and one normal (in the circle) subjects were selected for exome sequencing.

The anthropometric measurements of, and biochemical analyses performed in, the selected three subjects are summarized in Table 2. The BMI of the obese subjects were 38.34 and $44.29 \mathrm{~kg} / \mathrm{m}^{2}$; thereby, they were classified as obese types II and III, respectively. The BMI of the normal subject was $<23 \mathrm{~kg} / \mathrm{m}^{2}$. The blood pressure, pulse, HbA1C, cholesterol, and triglyceride levels in all three subjects were normal. The thickness of the triceps and subscapular skin fold of the obese subjects was twice that of the normal subject. DNA was extracted from peripheral leukocytes (concentration range $=300-1600 \mathrm{ng} / \mu \mathrm{L}$ ). The DNA purity, as determined by the absorbance ratios $\mathrm{A}_{260} / \mathrm{A}_{280}$ and $\mathrm{A}_{260} / \mathrm{A}_{230}$, was in the range of 1.71.8 and $1.9-2.2$, respectively. 
Table 2. Anthropometric measurements and results of biochemical analysis.

\begin{tabular}{|c|c|c|c|}
\hline Measurement & Ob1_6 & Ob1_15 & Ob1_7 \\
\hline Status & Obese Type III & Obese Type II & Normal \\
\hline Age (years) & 21 & 8 & 12 \\
\hline Gender & $\mathrm{F}$ & $\mathrm{M}$ & $\mathrm{M}$ \\
\hline Weight $(\mathrm{kg})$ & 119.85 & 84.55 & 53 \\
\hline Height $(\mathrm{m})$ & 164.5 & 148.5 & 152 \\
\hline BMI & 44.29 & 38.34 & 22.94 \\
\hline Blood pressure $(\mathrm{mmHg})$ & $116 / 67$ & $113 / 67$ & $109 / 70$ \\
\hline Pulse (bpm) & 61 & 96 & 82 \\
\hline Waist circumference $(\mathrm{cm})$ & 122.2 & 107.8 & 80.2 \\
\hline Hip circumference $(\mathrm{cm})$ & 127.1 & 106.3 & 86 \\
\hline $\mathrm{W} / \mathrm{H}$ ratio & 0.96 & 1.01 & 0.93 \\
\hline Triceps skinfold thickness (mm) & 48 & 33 & 19.4 \\
\hline Subscapular skinfold thickness $(\mathrm{mm})$ & 41.5 & 38.8 & 19 \\
\hline Total body fat percentage & 45.6 & NA & 22.3 \\
\hline Glucose $(\mathrm{mg} / \mathrm{dL})$ & 83 & 91 & 103 \\
\hline HbA1C (\%) & 5.6 & 5.9 & 5.5 \\
\hline Cholesterol (mg/dL) & 186 & 109 & 182 \\
\hline Insulin $(\mu \mathrm{U} / \mathrm{mL})$ & 11.12 & 20.71 & 70.59 \\
\hline Triglyceride $(\mathrm{mg} / \mathrm{dL})$ & 81 & 75 & 81 \\
\hline LDL-C (mg/dL) & 126.8 & 61 & 105.8 \\
\hline HDL-C (mg/dL) & 43 & 33 & 60 \\
\hline
\end{tabular}

LDL-C = low-density lipopolysaccharide cholesterol; HDL-C = high-density lipopolysaccharide cholesterol.

The workflow of exome sequencing is summarized in Figure 1. A thorough examination of the selected individuals yielded approximately 71,000 variants each. The quality of the obtained results was determined by the mean coverage per individual and the average total depth $(90 \%$ of the target region and 49 , respectively). Both obese subjects expressed 7201 variants, including 42 downstream variants, 4 frameshift deletions, 5 frameshift insertions, 17 non-frameshift deletions, and 10 non-frameshift insertions, and 639 nonsynonymous, 824 synonymous, 4 stop_gain, 44 unknown, 270 intergenic, 4493 intronic, 174 ncRNA, 118 upstream, 7 upstream or downstream, 13 splicing, 304 3'-untranslated region (UTR), and 233 5'-UTR variants, which were not observed in the normal individual (1 Table). Among these, 709 functional variants (nonsynonymous, frameshift_insertion, frameshift_deletion, stop gain, stop_loss, splicing, and unknown) were selected for further analyses.

The possible effect of these 709 variants on the structural and functional proteins was determined using PolyPhen-2, SIFT, and VarioWatch. The minimum depth of these 709 variants was approximately 20 , calculated as means \pm standard deviation (SD) of all values. Variants with a total depth $\geq 20$ were chosen: the total depth of 65 variants ( 60 genes) predicted to be deleterious by all three programs was $\geq 20$; these were selected for further analyses (Figure 1). All damaging variants uploaded to the UniProtKB database have been known to affect the molecular function. These variants included 52 single nucleotide variants ( 48 genes), 9 indel variants ( 9 genes), and 4 stop variants (4 genes). The 65 deleterious variants ( 60 genes) are summarized in $\underline{\mathbf{S 2} \text { Table. }}$.

Of the 9 indel variants, three insertion variants in the ALOX5AP, HCRTR1, and $R X F P 2$ genes were found in the splicing region. Two deletion variants in the DEFB126 and TIGD6 (rs3832324) genes and one insertion variant in GDPD4 (rs71272231) were found in the exonic region; however, these were classified as frameshift deletion and frameshift insertion, respectively. The variants occurring in the OPLAH (rs141499204) and OR4C45 genes, also in the exonic region, were classified as unknown variants. The insertion variant in $M L L 3$ was identified as a stop codon. 


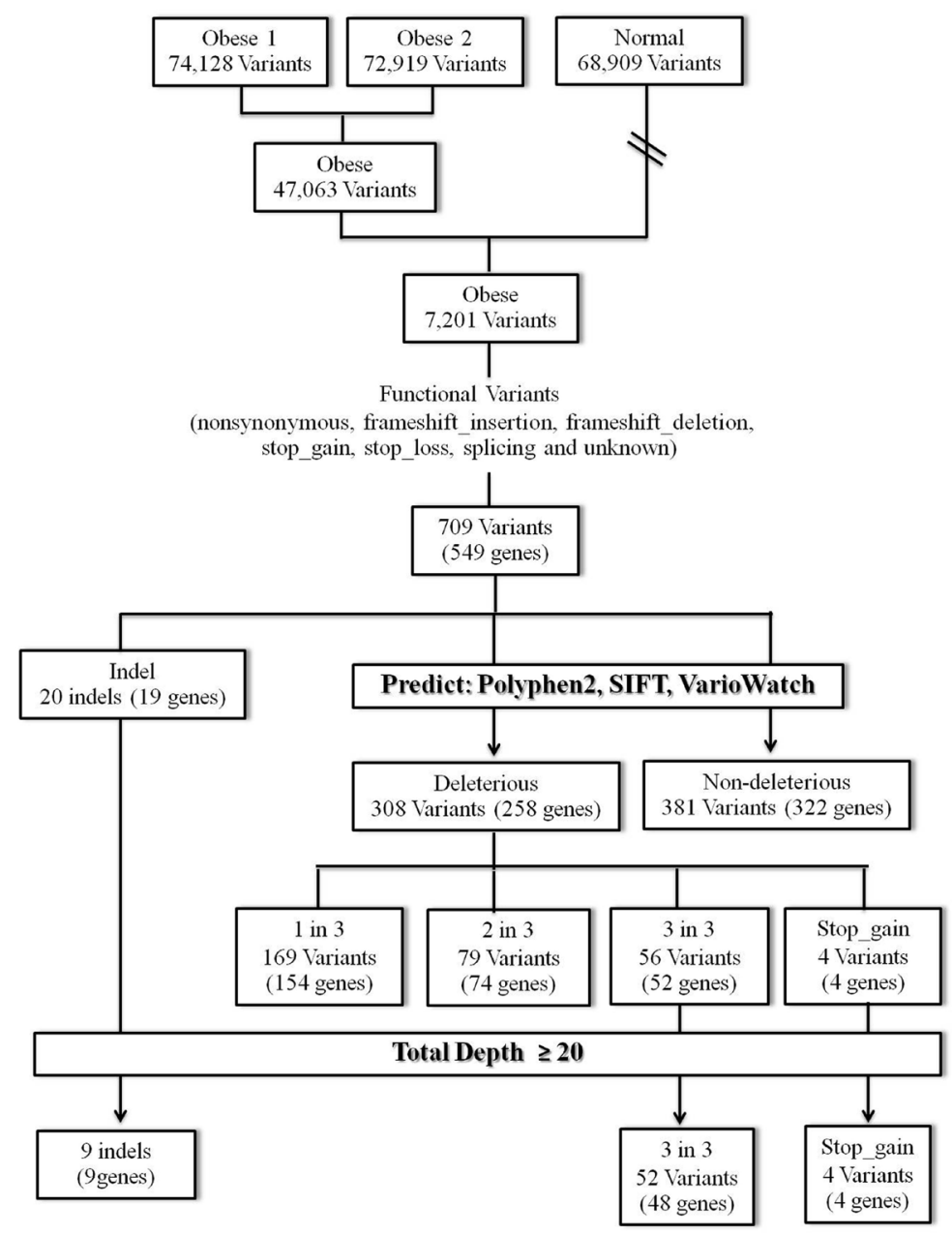

Figure 1. Workflow of identification of causal variants related to obesity traits.

Four variants wherein a nucleotide change resulted in a stop codon were observed in the COL9A2 (rs12077871), MS4A12 (rs2298553), PDE4DIP (rs2762779), and PRIM2 (rs62398999) genes.

The possible pathways of the 60 genes expressing the 65 deleterious variants were predicted using the Reactome Pathway Browser, KEGG pathway database, and pathcards ${ }^{\text {alpha }}$ tool in the GeneCards ${ }^{\circledR}$ website (Table 3 and $\underline{\mathbf{S 3} \text { Table) }}$. These genes were categorized into 13 groups, based on their possible functions in signal transduction, the metabolic (lipid, protein, or glutathione) pathways, the immune system, disease [specifically, the ACTH deficiency and pro-opiomelanocortinin deficiency (POMCD)] pathways, transmembrane transport of small molecules, developmental biology, extracellular matrix organization, gene expression, cell cycle, chromatin organization, DNA replication, homeostasis, and membrane trafficking. A majority of these genes were involved in the signal transduction pathway. 
Table 3. Possible functional pathways of the 60 selected genes.

\begin{tabular}{l|c|l}
\hline Possible pathway & Gene number & Gene \\
\hline Signal transduction & 16 & $\begin{array}{l}\text { ARHGEF3, CARD14, COL4A4, COL9A2, HCRTR1, MS4A12, OR10H5, OR10Z1, } \\
\text { OR11H6, OR4C45, OR4K15, OR5AU1, OR8D1, PDE4DIP, RGL3, RXFP2 }\end{array}$ \\
\hline Gene expression & 10 & $\begin{array}{l}\text { KIAA0564, MTERFD2, PNN, SRBD1, TIGD6, TTC22, ZNF35, ZNF140, ZNF474, } \\
\text { ZNF596 }\end{array}$ \\
\hline Chromatin organization & 8 & LRGUK, MLL3, SMYD4, TGM4, USP49, ZNF35, ZNF474, ZNF596 \\
\hline Metabolism & 7 & ALDH1B1, ALOX5AP, CYP4A22, GDPD4, MUC13, OPLAH, PLA2G3 \\
\hline - Lipid metabolism & & ALDH1B1, ALOX5AP, CYP4A22, GDPD4, PLA2G3 \\
\hline - Protein metabolism & & MUC13 \\
\hline - Glutathione metabolism & 6 & OPLAH \\
\hline Immune system & 5 & COLI3L1, DEFB126, COLAPA2, GPR98, SPAG17, SPTB \\
\hline Developmental biology & 4 & CYP4A22, HCRTR1, OPLAH, RXFP2 \\
\hline Disease & 5 & ABCA6, SLC29A3, SLC9A10, TRPM8, GPR172B \\
\hline Transmembrane transport of small molecules & 4 & C11orf34, COL4A4, COL9A2, ITGAE \\
\hline Extracellular matrix organization & 4 & DOCK6, FREM2, MKI67, PCDHGA8 \\
\hline Hemostasis & 2 & PRIM2, ZNF596 \\
\hline Cell cycle & 2 & PRIM2, TLR3 \\
\hline DNA replication & 1 & $A S P S C R 1$ \\
\hline Membrane trafficking & &
\end{tabular}

A low- or rare-minor allele frequency (MAF; $0.5 \leq \mathrm{MAF}<5 \%$ and $\mathrm{MAF}<0.5 \%$, respectively) could clarify the association between these variants and complex diseases or traits. Evolutionary theory suggests that the frequency of these deleterious variants could be rare (Lee et al., 2014). The MAF of variants in COL9A2 and TRPM8 was found to be $<0.5 \%$. Moreover, the rs 12077871 variant in COL9A2 was found to be a stop codon at position 326 of the amino acid sequence. The MAF of 12 variants in the ALOX5AP, DEFB126, GDPD4, HCRTR1, MLL3, OPLAH, OR4C45, PRIM2, RXFP2, TIGD6, USP49, and ZNF596 genes were not reported in the 1000 genome database.

Six genes with a low or rare MAF, predicted to be associated with control feeding behavior and lipid metabolism by Reactome Pathway Browser and KEGG pathway database analysis, were chosen for further genotyping in the members of this family. The normal subjects did not express the A allele in the COL9A2 gene and the homozygous variant genotype CC in TRPM8; however, this difference between the analyzed normal and obese individuals was not statistically significant $(\mathrm{P}=0.261$ and 0.715 , respectively; Figure 2$)$. However, when the genotype percentage was adjusted, we observed that variants in HCRTR1, COL9A2, and TRPM8 were significantly different between obese and normal subjects $\left(\mathrm{P}=3.3 \times 10^{-5}, 7.6 \times 10^{-5}\right.$, and 0.0262 , respectively).



Figure 2. Percentage genotype frequency of the 6 selected genes in this family. 


\section{DISCUSSION}

GWAS are useful tools, which can be used to identify common variants associated with complex diseases. Loci associated with increased BMI in adults by GWAS are also found in cases of childhood obesity (den Hoed et al., 2010). A majority of the reported obesity genetic variants are shared among Europeans, African-Americans, South Asians, and East Asians (Hotta et al., 2009; Bollepalli et al., 2010; Croteau-Chonka et al., 2011; Hester et al., 2012). A few loci near the genes PP13439-TMEM212, CDH12, MFAP3-GALNT10, and FER1L4 were also associated with the BMI in an African-American population (Ng et al., 2012). Variants of LEP, LEPR, POMC, PCSK1, FTO, MC3R, MC4R, GNPDA2, TMEM18, QPCTL/GIPR, BDNF, ETV5, MAP2K5/SKOR1, SEC16B, SIM1, and TNKS/MSRA that were suspected to be associated with obesity and obesity-related traits were identified from among a massive amount of genome data. However, a majority of these variants were in the intronic or intergenic region (Dorajoo et al., 2012; Loos, 2012; Chuenta et al., 2015). Thus, the mechanisms of these phenotypes cannot be explained.

Exome sequencing has been used in the precise identification of causal gene variants leading to disease, as only coding regions of the genes are detected. This is the first study to use the exome sequencing approach to identify exonic variant(s) related to the BMI in an obese Thai family. The expression of variants of genes identified by GWAS, including $L E P, L E P R, P O M C$, PCSK1, FTO, MC3R, MC4R, GNPDA2, TMEM18, QPCTL/GIPR, BDNF, ETV5, MAP2K5/ SKOR1, SEC16B, SIM1, and TNKS/MSRA (Farooqi et al., 2003; Begriche et al., 2011; Dubern and Clement, 2012; Loos, 2012; Mul et al., 2012; Tsou et al., 2012), did not differ significantly between obese and normal subjects. Subsequently, 65 variants in 60 genes predicted to negatively affect the protein structure and function were chosen for further analyses.

Low- and rare-MAF variants are generally associated with complex traits or diseases (Tennessen et al., 2012; Lee et al., 2014); therefore, these deleterious variants were also surmised to express a low or rare MAF (Lee et al., 2014). Fourteen genes with an MAF $<5 \%$ (ALOX5AP, COL9A2, DEFB126, GDPD4, HCRTR1, MLL3, OPLAH, OR4C45, PRIM2, RXFP2, TIGD6, TRPM8, USP49, and ZNF596) were analyzed to identify a possible obesity trait or disease risk. Reactome Pathway Browser and KEGG pathway database analyses revealed that these genes are involved in a network of pathways, including lipid metabolism, signaling transduction, immune, membrane transport, and gene regulatory pathways, in complex diseases such as obesity.

Consumption of a high fat diet leads to the accumulation of fat, and subsequent formation of adipose tissue, in the visceral organs and skeletal muscles. Adipose tissue comprises preadipocytes, adipocytes, adipose tissue macrophages, fibroblasts, endothelial cells, collagenous tissue, and mesenchymal stem cells (Toda et al., 2009; Rezaee and Dashty, 2013). Activated adipose tissue macrophages release pro-inflammatory cytokines such as tumor necrosis factor (TNF)- $\alpha$, interleukin (IL)-6, IL-1, and monocyte chemoattractant protein (MCP)-1; these factors promote lipolysis and secrete free fatty acids (FFA) into the circulating blood (Cartier, 2010; Kawasaki et al., 2012; Samad and Ruf, 2013). Hypoxia, caused by obstructive sleep apnea, is another factor inducing lipolysis and the generation of FFA. Excess FFA increases $\beta$-oxidation and reactive oxygen species production, which consequently promotes organelle toxicity and apoptosis (Chiang et al., 2011; Kawasaki et al., 2012). Additionally, this induces endoplasmic reticulum stress, mitochondrial dysfunction, systemic inflammation, development of arterial or venal thrombosis, and fatty acid-induced insulin pathway disturbance (which 
is involved in the pathogenesis of insulin resistance), development of arterial thrombosis, cardiovascular disease and diabetes (through the upregulation of Fas), activation of JNK, and destabilization of lysosomes (Cartier, 2010; Rezaee and Dashty, 2013; Samad and Ruf, 2013). These pro-inflammatory cytokines affect the blood vasculature or the liver, thereby increasing the risk of cardiovascular disease and diabetes. They also impact the signal transduction pathway in the brain, especially in the hypothalamus, which affects the regulation of food intake. Adipose tissues also play a significant role in the regulation of the metabolic system, leading to the induction of metabolic disease.

The imbalance between adipokines (such as adiponectin, leptin, and resistin) and inflammatory cytokines (such as TNF- $\alpha$, IL-6, IL-1, and MCP-1) in addition to insulin resistance promotes liver injury in hepatic steatosis. Transdifferentiation of hepatic stellate cells into contractile myofibroblasts and remodeling of the extracellular matrix are the two major mechanisms that contribute to hepatic wound healing (and subsequently the development of fibrosis) in acute liver injury. Subsequently, collagen is deposited on the remodeled hepatocyte, which might cause liver dysfunction and is a risk factor of non-alcoholic fatty liver disease (Chiang et al., 2011).

Obesity and the production of inflammatory cytokines are risk factors for the development of arterial or venal thrombosis. Pro-inflammatory cytokines are known to react with the cell surface of tissue factors (TFs) via protein disulfide isomerase-dependent thioldisulfide exchange. At high levels of expression; this leads to an increase in the level of bloodborne $\mathrm{TF}$ in the vascular compartment and induces the upregulation of transcription factors as well as mRNA splicing (Samad and Ruf, 2013). TF is found in many organs, including the skin, epithelia of the gastrointestinal tract, uterus, placenta, brain, lungs, smooth muscle, and blood vessels. Atherosclerotic plaques are known to accumulate in the cerebral or coronary vessels, resulting in thrombosis via a coagulation-signaling cascade, which subsequently develops into an atherosclerotic cardiovascular disease (Jackson, 2011). High expressions of $A L O X 5 A P$ is associated with increased body weight, homeostatic model assessment of insulin resistance (HOMA-IR), and atherosclerotic cardiovascular disease, but not with obesity and insulin resistance (Kaaman et al., 2006). In this study, obese subjects showed a higher rate of AT insertion at the splice site of exon 1 in $A L O X 5 A P$, involved in the development of inflammatory adipose tissue; however, this difference was not statistically significant.

A previous study has observed an increase in the inflammatory signaling activity in the brain in response to overnutrition (Hummasti and Hotamisligil, 2010). Overnutritionrelated metabolic inflammation in the hypothalamus, specifically in the POMC neurons, was found to be associated with the development of hypertension in obese mice (Purkayastha et al., 2011). In this study, the signal transducing genes HCRTR1, OR4C45, and RXFP2, involved in neuroactive ligand-receptor interaction in the hypothalamus, were found to undergo polymorphisms, affecting the regulation of food intake (Kong et al., 2010; Flores et al., 2013). HCRTR1 gene encodes hypocretin receptor type 1, a protein belonging to the G-protein coupled receptor family, which is involved in the regulation of feeding behavior and energy homeostasis. This protein also binds with neuropeptide orexin A, which is located in the hypothalamus; the orexin peptide stimulates wakefulness and energy expenditure (Flier and Maratos-Flier, 1998). Moreover, orexin A stimulates glucose uptake while inhibiting lipolysis (Skrzypski et al., 2011). Previous studies have reported that orexin deficiency leads to narcolepsy in humans; this results in an energy imbalance due to decreased energy intake and increase in BMI, which in turn increases the risk of obesity (Sakurai, 2005). RXFP2 encodes 
a G-protein coupled receptor subfamily containing a leucine-repeat region. $R X F P 2$ increases the accumulation of cAMP in a ligand-independent manner; therefore, this receptor plays a role in the Gs-cAMP-protein kinase A pathway, as well as the melanocortin pathway. Defects in this gene induce a decrease in cAMP accumulation, leading to a decrease in energy expenditure. In this study, obese subjects showed a significant increase in the rate of CCT insertion at the end of exon 9 in HCRTR 1 and AAA insertion at exon 12 in RXFP2 compared to the normal subjects.

Interestingly, the results of exome sequencing highlighted the TRPM8 gene, which involves in activated brown adipose tissue (BAT), inducing energy expenditure and weight loss. The administration of $\beta$-adrenergic receptor agonists or chemical uncoupling agents are sensed by the transient receptor potential family of cation channels, such as TRPAI and TRPM8, because of the low temperatures. The cold receptor TRPM8 signals various regions of the brain, including the paraventricular hypothalamus, arcuate nucleus, lateral hypothalamus, and ventromedial hypothalamus, resulting in the regulation of uncoupling protein 1. BATs, which are activated, then utilize energy (Chechi et al., 2013). In this study, obese subjects displayed a mutation in exon 7 of TRPM8, inducing the expression of the $\mathrm{C}$ allele, resulting in an arginine $\rightarrow$ threonine and positive charge $\rightarrow$ uncharged mutations.

In conclusion, exome sequencing was performed to identify variants in the coding regions of genes associated with obesity and obesity-related traits in a Thai consanguineous family. This method could be used to identify causal genes and possible pathways associated with obesity and obesity-related traits. We conclude that obesity may be regulated by a polygenic pattern, and controlled by several pathways that regulate the feeding behavior and energy expenditure, in this family.

\section{Conflicts of interest}

The authors declare no conflict of interest.

\section{ACKNOWLEDGMENTS}

We are grateful to all the participants of this study. We would also like to thank the staff at the Department of Tropical Nutrition and Food Science, Faculty of Tropical Medicine; the Department of Pediatrics, Faculty of Medicine Siriraj Hospital; and the Department of Pediatrics, Faculty of Medicine Ramathibodi Hospital, Mahidol University, for their support. Research supported by the Nation Research of Council of Thailand grant (NRCT; \#2557A11002004).

\section{REFERENCES}

Begriche K, Levasseur PR, Zhang J, Rossi J, et al. (2011). Genetic dissection of the functions of the melanocortin-3 receptor, a seven-transmembrane G-protein-coupled receptor, suggests roles for central and peripheral receptors in energy homeostasis. J. Biol. Chem. 286: 40771-40781. http://dx.doi.org/10.1074/jbc.M111.278374

Bollepalli S, Dolan LM, Deka R and Martin LJ (2010). Association of FTO gene variants with adiposity in AfricanAmerican adolescents. Obesity (Silver Spring) 18: 1959-1963. http://dx.doi.org/10.1038/oby.2010.82

Cartier A (2010). The inflammatory profile associated with abdominal obesity. Official J. Int. Chair on Cardiomet. Risk 3: $15-19$.

Chechi K, Carpentier AC and Richard D (2013). Understanding the brown adipocyte as a contributor to energy homeostasis. Trends Endocrinol. Metab. 24: 408-420. http://dx.doi.org/10.1016/j.tem.2013.04.002 
Chiang DJ, Pritchard MT and Nagy LE (2011). Obesity, diabetes mellitus, and liver fibrosis. Am. J. Physiol. Gastrointest. Liver Physiol. 300: G697-G702. http://dx.doi.org/10.1152/ajpgi.00426.2010

Chuenta W, Phonrat B, Tungtrongchitr A, Limwongse C, et al. (2015). Common variations in the FTO gene and obesity in Thais: a family-based study. Gene 558: 75-81. http://dx.doi.org/10.1016/j.gene.2014.12.050

Croteau-Chonka DC, Marvelle AF, Lange EM, Lee NR, et al. (2011). Genome-wide association study of anthropometric traits and evidence of interactions with age and study year in Filipino women. Obesity (Silver Spring) 19: 1019-1027. http://dx.doi.org/10.1038/oby.2010.256

Davis L, Dibner M and Battey J (1986). Rapid DNA preparation. In: Basic methods in molecular biology (Davis L, eds.). Elsevier, New York, 43.

de Onis M, Blössner M and Borghi E (2010). Global prevalence and trends of overweight and obesity among preschool children. Am. J. Clin. Nutr. 92: 1257-1264. http://dx.doi.org/10.3945/ajen.2010.29786

den Hoed M, Ekelund U, Brage S, Grontved A, et al. (2010). Genetic susceptibility to obesity and related traits in childhood and adolescence: influence of loci identified by genome-wide association studies. Diabetes 59: 2980-2988. http:// dx.doi.org/10.2337/db10-0370

Dorajoo R, Blakemore AI, Sim X, Ong RT, et al. (2012). Replication of 13 obesity loci among Singaporean Chinese, Malay and Asian-Indian populations. Int. J. Obes. 36: 159-163. http://dx.doi.org/10.1038/ijo.2011.86

Dubern B and Clement K (2012). Leptin and leptin receptor-related monogenic obesity. Biochimie 94: 2111-2115. http:// dx.doi.org/10.1016/j.biochi.2012.05.010

Farooqi IS, Keogh JM, Yeo GS, Lank EJ, et al. (2003). Clinical spectrum of obesity and mutations in the melanocortin 4 receptor gene. N. Engl. J. Med. 348: 1085-1095. http://dx.doi.org/10.1056/NEJMoa022050

Flier JS and Maratos-Flier E (1998). Obesity and the hypothalamus: novel peptides for new pathways. Cell 92: 437-440. http://dx.doi.org/10.1016/S0092-8674(00)80937-X

Flores A, Maldonado R and Berrendero F (2013). Cannabinoid-hypocretin cross-talk in the central nervous system: what we know so far. Front. Neurosci. 7: 256. http://dx.doi.org/10.3389/fnins.2013.00256

Haslam DW and James WP (2005). Obesity. Lancet 366: 1197-1209. http://dx.doi.org/10.1016/S0140-6736(05)67483-1

Hester JM, Wing MR, Li J, Palmer ND, et al. (2012). Implication of European-derived adiposity loci in African Americans. Int. J. Obes. 36: 465-473. http://dx.doi.org/10.1038/ijo.2011.131

Hotta K, Nakamura M, Nakamura T, Matsuo T, et al. (2009). Association between obesity and polymorphisms in SEC16B, TMEM18, GNPDA2, BDNF, FAIM2 and MC4R in a Japanese population. J. Hum. Genet. 54: 727-731. http:// dx.doi.org/10.1038/jhg.2009.106

Hummasti S and Hotamisligil GS (2010). Endoplasmic reticulum stress and inflammation in obesity and diabetes. Circ. Res. 107: 579-591. http://dx.doi.org/10.1161/CIRCRESAHA.110.225698

Jackson SP (2011). Arterial thrombosis--insidious, unpredictable and deadly. Nat. Med. 17: 1423-1436. http://dx.doi. org $/ 10.1038 / \mathrm{nm} .2515$

Kaaman M, Rydén M, Axelsson T, Nordström E, et al. (2006). ALOX5AP expression, but not gene haplotypes, is associated with obesity and insulin resistance. Int. J. Obes. 30: 447-452. http://dx.doi.org/10.1038/sj.ijo.0803147

Kawasaki N, Asada R, Saito A, Kanemoto S, et al. (2012). Obesity-induced endoplasmic reticulum stress causes chronic inflammation in adipose tissue. Sci. Rep. 2: 799. http://dx.doi.org/10.1038/srep00799

Kong RC, Shilling PJ, Lobb DK, Gooley PR, et al. (2010). Membrane receptors: structure and function of the relaxin family peptide receptors. Mol. Cell. Endocrinol. 320: 1-15. http://dx.doi.org/10.1016/j.mce.2010.02.003

Lee S, Abecasis GR, Boehnke M and Lin X (2014). Rare-variant association analysis: study designs and statistical tests. Am. J. Hum. Genet. 95: 5-23. http://dx.doi.org/10.1016/j.ajhg.2014.06.009

Loos RJ (2012). Genetic determinants of common obesity and their value in prediction. Best Pract. Res. Clin. Endocrinol. Metab. 26: 211-226. http://dx.doi.org/10.1016/j.beem.2011.11.003

Maffeis C and Tatò L (2001). Long-term effects of childhood obesity on morbidity and mortality. Horm. Res. 55 (Suppl 1): 42-45. http://dx.doi.org/10.1159/000063462

Mul JD, van Boxtel R, Bergen DJ, Brans MA, et al. (2012). Melanocortin receptor 4 deficiency affects body weight regulation, grooming behavior, and substrate preference in the rat. Obesity (Silver Spring) 20: 612-621. http://dx.doi. org/10.1038/oby.2011.81

$\mathrm{Ng}$ MC, Hester JM, Wing MR, Li J, et al. (2012). Genome-wide association of BMI in African Americans. Obesity (Silver Spring) 20: 622-627. http://dx.doi.org/10.1038/oby.2011.154

Purkayastha S, Zhang G and Cai D (2011). Uncoupling the mechanisms of obesity and hypertension by targeting hypothalamic IKK-b and NF-kB. Nat. Med. 17: 883-887. http://dx.doi.org/10.1038/nm.2372

Rezaee F and Dashty M (2013). Role of adipose tissue in metabolic system disorders adipose tissue is the initiator of metabolic diseases. J. Diabetes Metab. S13: 1-9. 
Sakurai T (2005). Roles of orexin/hypocretin in regulation of sleep/wakefulness and energy homeostasis. Sleep Med. Rev. 9: 231-241. http://dx.doi.org/10.1016/j.smrv.2004.07.007

Samad F and Ruf W (2013). Inflammation, obesity, and thrombosis. Blood 122: 3415-3422. http://dx.doi.org/10.1182/ blood-2013-05-427708

Skrzypski M, T Le T, Kaczmarek P, Pruszynska-Oszmalek E, et al. (2011). Orexin A stimulates glucose uptake, lipid accumulation and adiponectin secretion from 3T3-L1 adipocytes and isolated primary rat adipocytes. Diabetologia 54: 1841-1852. http://dx.doi.org/10.1007/s00125-011-2152-2

Tennessen JA, Bigham AW, O'Connor TD, Fu W, et al.; Broad GO; Seattle GO; NHLBI Exome Sequencing Project (2012). Evolution and functional impact of rare coding variation from deep sequencing of human exomes. Science 337: 64-69. http://dx.doi.org/10.1126/science. 1219240

Toda S, Uchihashi K, Aoki S, Sonoda E, et al. (2009). Adipose tissue-organotypic culture system as a promising model for studying adipose tissue biology and regeneration. Organogenesis 5: 50-56. http://dx.doi.org/10.4161/org.5.2.8347.

Tsou RC, Zimmer DJ, De Jonghe BC and Bence KK (2012). Deficiency of PTP1B in leptin receptor-expressing neurons leads to decreased body weight and adiposity in mice. Endocrinology 153: 4227-4237. http://dx.doi.org/10.1210/ en.2012-1548

\section{Supplementary material}

S1 Figure. Pedigree diagram of the study family.

S1 Table. Summary of the 7201 variants observed in both obese subjects.

S2 Table. Details of the 65 deleterious variants and their effects on protein structure and function.

S3 Table. Predicted possible pathways of the 60 genes using Reactome Pathway Browser, KEGG pathway database, and pathcards alpha tool in the GeneCards ${ }^{\circledR}$ website. 